\title{
Identification and Molecular Characterization of Castellaniella ginsengisoli Isolated from Sugarcane-Wheat Cropping System
}

\author{
Priyanka Chandra ${ }^{1}$ and Amaresh Chandra ${ }^{2 *}$ \\ ${ }^{1}$ ICAR - Central Soil Salinity Research Institute, Karnal, Haryana, India \\ ${ }^{2}$ ICAR - Indian Institute of Sugarcane Research, Lucknow, U.P., India \\ *Corresponding author
}

\section{A B S T R A C T}

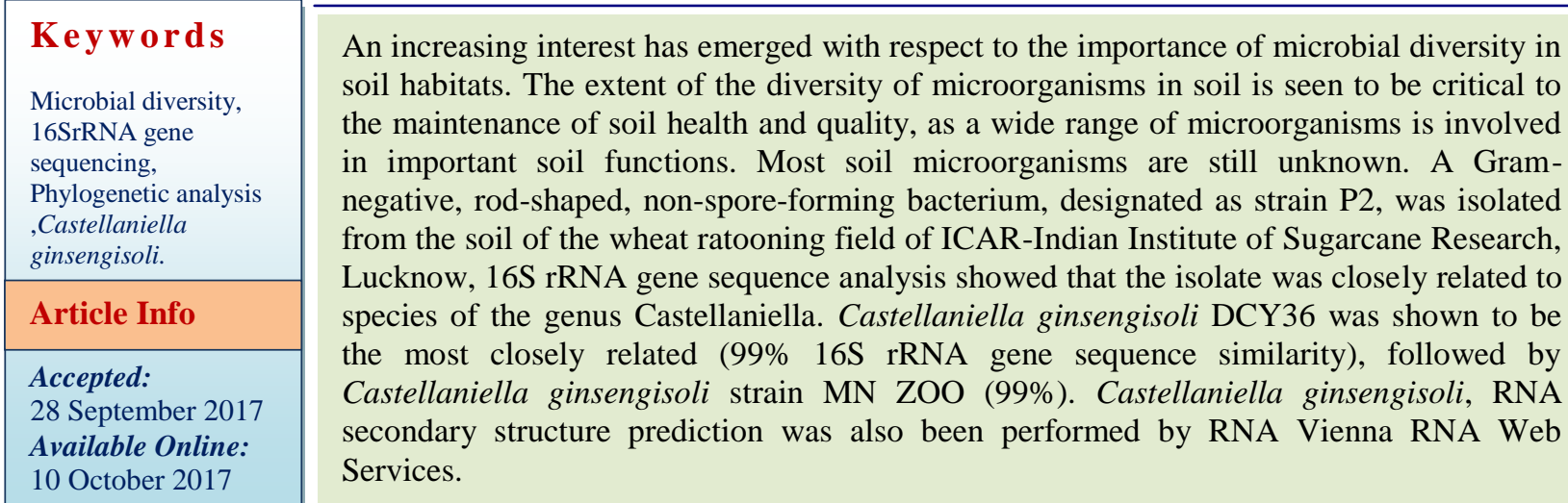

\section{Introduction}

Soils typically contain $10^{9}$ to $10^{10}$ microorganisms per gram (dry weight), which may represent more than a million bacterial species. However, characterization of the small fraction of microbes that has been cultivated provides only a glimpse of their potential physiological capacity and influence on soil ecosystems. Soil is considered to be the richest environment, with a high diversity of microorganisms belonging to the three domains of life, Bacteria, Archaea and Eukarya. This diversity is extreme at the species level, with approximately 50,000 bacterial species found in one soil sample. Investigation of bacterial diversity is an important step to assess soil conditions due to its importance in nutrient cycling, and consequently in crop productivity. Soil bacteria and, in particular, rhizosphere bacteria play an important role in many processes, such as decomposition, mineralization, biological nitrogen fixation, and denitrification. In addition, some bacteria associate with plants and promote growth, the so-called plant growth-promoting bacteria (Singh et al., 2004). In the course of screening micro-organisms obtained from the soil of sugarcane-wheat cropping system, a Gramnegative strain, P2, was isolated. The following study was carried out for the identification of the bacteria by 16s RNA techniques. 


\section{Materials and Methods}

The soil samples used for isolation of bacteria was collected from the root-free soil of rhizosphere from after wheat ratooning field of ICAR-Indian Institute of Sugarcane Research, Lucknow. The sample of each varietal rhizosphere soil was mixed thoroughly to make a composite soil. $10 \mathrm{~g}$ of dry and highly pulvirised soil sample is suspended in $90 \mathrm{ml}$ of sterile distilled water considered as a stock solution then transferring $1 \mathrm{ml}$ of soil suspension into $9 \mathrm{ml}$ sterile distilled water with the help of a sterile pipette to yield 10 dilution. Similarly, a series up 1 to 10 dilution was prepared under aseptic condition. Bacteria are isolated by employing serial dilution plate technique using nutrient agar. Then $0.1 \mathrm{ml}$ soil suspension is introduced into sterilized nutrient agar media in Petri dishes and spread it thoroughly on the media incubated at $37^{\circ} \mathrm{C}$ for $24-48$ hours and for each dilution the plates are taken in triplicates. After incubation period, visual morphological characterization of the bacterial colonies isolated on the agar petri plates is observed on the basis of colour, shape, size, elevation etc. of the bacterial colonies. Colonies exhibiting prolific growth are selected for further streaking on fresh agar plates for purification and multiplication of the isolates is done by streak plate methods.

The isolate was grown on nutrient agar plates at $37^{\circ} \mathrm{C}$ for $24-48 \mathrm{~h}$ and was maintained on nutrient agar slants and stored at $4^{\circ} \mathrm{C}$ as well as at $-80^{\circ} \mathrm{C}$ by making their suspensions in $10 \%(\mathrm{v} / \mathrm{v})$ glycerol.

Identification of bacterial strain was done using 16SrRNA gene sequencing. The DNA template was prepared by picking an individual colony of bacterial strain , and amplification of the 16S rRNA gene was carried out by the PCR . PCR amplification of DNA was performed using universal primers (9F: 5'-GAGTTTGATCCTGGC TCAG -3';
1510R: 5'-GGCTACCTTGTTACGA-3') in a reaction mixture $(25 \mu \mathrm{l})$. The amplification program for the full-length 16S rRNA gene consisted of an initial denaturion at $94{ }^{\circ} \mathrm{C}$ for 2 min, followed by 30 cycles of denaturation at $94{ }^{\circ} \mathrm{C}$ for $2 \mathrm{~min}$, primer annealing at $55^{\circ} \mathrm{C}$ for $1 \mathrm{~min}$ and primer extension at $72{ }^{\circ} \mathrm{C}$ for 2 min, followed by a final extension at $72{ }^{\circ} \mathrm{C}$ for $10 \mathrm{~min}$, in a thermocycler. Amplified PCR products of the $16 \mathrm{~S}$ ribosomal gene were separated on $1 \%$ agarose gel in $0.5 \times \mathrm{TE}$ (Tris-EDTA) buffer containing $2 \mu 1$ ethidium bromide $(20 \mathrm{mg} / \mathrm{ml})$ (Chandra and Chandra, 2016). The purified PCR product samples were sent for sequencing using universal $16 \mathrm{~S}$ rRNA sequencing primers.

The sequence results were obtained from a BLAST search, and the sequences of all the related species were retrieved to determine the exact nomenclature of the isolates. The tree is created using Weighbor with alphabet size 4 and length size 1000 .

\section{Results and Discussion}

The ribosomal operons mainly $16 \mathrm{~S}$ rRNA has proven to be a stable and specific molecular marker for the identification of bacteria. The copy number of $16 \mathrm{~S}$ rDNA genes may fluctuate from 1 to 15 among different bacterial genomes. The $16 \mathrm{~S}$ rDNA is present in scattered form in the entire genome of bacteria. These ribosomal sequences are useful for the phylogenetic analysis and molecular taxonomy of bacteria. The $16 \mathrm{~S}$ rDNA is a common target for the taxonomical purpose, mainly due to the mosaic composition of phylogenetically conserved and variable region within the gene (Pontes $e t$ al., 2007). The aligned sequence data of isolate was 1382bp. 16S rRNA gene sequences were compared with the available sequences in the databank with help of BLAST homology search and the isolate was found to be Castellaniella ginsengisoli (Figure 1). Homology tree based on sequence 
alignment of 16S rDNA of bacterial isolates permitted rapid phylogenetic analysis. However, strains isolated from different geographic location shared similar DNA homology. Phylogenetic analysis on the basis of $16 \mathrm{~S}$ rDNA sequences provided better understanding in evaluation of genetic diversity of bacteria isolated from same and different ecological niche; phylogenetic analysis of $500 \mathrm{bp}$ of terminal region of $16 \mathrm{~S}$ rDNA from cultivated strain has been found to show existence of large bacterial diversity. A phylogenetic tree or evolutionary tree is a branching diagram or tree showing the inferred evolutionary relationships among various biological species or other entities based upon similarities and differences in their physical and/or genetic characteristics. The taxa joined together in the tree are implied to have descended from a common ancestor. Unrooted trees illustrate the relatedness of the leaf nodes without making assumptions about ancestry at all. In the case of unrooted trees, branching relationships between taxa are specified by the way they are connected to each other, but the position of the common ancestor is not (Mooers and Heard, 2004). The Microbe was found to be most similar Castellaniella ginsengisoli strain DCY36 16S ribosomal RNA gene, partial sequence Sequence ID: ref|NR_116482.1 and the next closest homologue was found to be Castellaniella ginsengisoli strain MNzoo 16S ribosomal RNA gene, complete sequence Sequence ID: $\mathrm{gb} \mid$ KM275476.1 (Table 1). The sequences of strain were submitted to NCBI Gene Bank database under accession numbers KY606683. The sequence is as follows:

AGTAAAAGGCTTGGCTTTCTTGGGCGG CGAGTGGCGAACGGGTGAGTAATGTA TCGGAACGTGCCCAGTAGCGGGGGAT AACTGGCCGAAAGGTCAGCTAATACC GCATACGCCCTACGGGGGAAAGGGGG GGATCGCAAGACCTCTCACTATTGGAG
CGGCCGATATCGGATTAGCTAGTTGGT GGGGTAAAGGCCTACCAAGGCAACGA TCCGTAGCTGGTTTGAGAGGACGACCA GCCACACTGGGACTGGACACGGCCCA GACTCCTACGGGAGGCAGCAGTGGGG AATTTTGGACAATGGGGGCAACCCTGA TCCAGCCATCCCGCGTGTGCGATGAAG GCCTTCGGGTTGTAAAGCACTTTTGGC AGGGAAGAAACAGCCCGGGCTAATAT CCCGGGTCAATGACGGTACCTGCAGA ATAAGCACCGGCTAACTACGTGCCAGC AGCCGCGGTAATACGTAGGGTGCAAG CGTTAATCGGAATTACTGGGCGTAAAG CGTGCGCAGGCGGTTCGGAAAGAAAG GTGTGAAATCCCAGGGCTTAACCTTGG AACTGCACTTTTAACTACCGGGCTAGA GTACGTCAGAGGGGGGTAGAATTCCA CGTGTAGCAGTGAAATGCGTAGAGAT GTGGAGGAATACCGATGGCGAAGGCA GCCCCCTGGGATGATACTGACGCTCAT GCACGAAAGCGTGGGGAGCAAACAGG ATTAGATACCCTGGTAGTCCACGCCCT AAACGATGTCAACTAGCTGTTGGGGTT TATTAACCTTAGTAGCGCAGCTAACGC GTGAAGTTGACCGCCTGGGGAGTACG GCGCAAGATTAAAACTCAAAGGAATT GACGGGGACCCGCACAAGCGGTGGAT GATGTGGATTAATTCGATGCAACGCGA AAAACCTTACCTACCCTTGACATGTCT GGAATCCTTTAGAGATAGAGGAGTGCT CGCAAGAGAACCGGAACACAGGTGCT GCATGGCTGTCGTCAGCTCGTGTCGTG AGATGTTGGGTTAAGTCCCGCAACGAG CGCAACCCTTGCCATTAGTTGCTACAT TCAGTTGGGCACTCTAATGGGACTGCC GGTGACAAACCGGAGGAAGGTGGGGA TGACGTCAAGTCCTCATGGCCCTTATG GGTAGGGCTTCACACGTCATACAATGG TCGGGACAGAGGGTTGCCAAACCGCG AGGTGGAGCCAATCTCAGAAACCCGA TCGTAGTCCGGATCGCAGTCTGCAACT CGACTGCGTGAAGTCGGAATCGCTAGT AATCGCGGATCAGCATGTCGCGGTGA ATACGTTCCCGGGTCTTGTACACACCG CCCGTCACACCATGGGAGTGGGTTTCA CCAGAAGTAGGTAGCCTAACACGACA 
Table.1 Table demonstrating percentage match between the bacteria with their accession no

\begin{tabular}{llll}
\hline S. No. & Organism Name & Accession No. & Percentage Match \\
\hline 1 & Castellaniella ginsengisoli strain DCY36 & NR_116 & $99 \%$ \\
2 & Castellaniella ginsengisoli strain MN ZOO & KM275476.1 & $99 \%$ \\
3 & Uncultured bacterium clone 7-5 & JQ923845.1 & $99 \%$ \\
4 & Castellaniella sp. Pyr2 & GU951457.1 & $99 \%$ \\
5 & Castellaniella sp. 528F1-2 & EU851054 & $99 \%$ \\
6 & Castellaniella sp. TCOB-5 & KU257691.1 & $99 \%$ \\
7 & Alcaligenes sp. A36 & KT316405.1 & $99 \%$ \\
8 & Alcaligenes sp. ZL3 & JN085953 & $99 \%$ \\
9 & Uncultured Alcaligenes sp. clone H2 & FJ863103.1 & $99 \%$ \\
10 & Beta proteobacterium C14 JRPA-2007 & EF599312.1 & $99 \%$ \\
\hline
\end{tabular}

Fig.1 Phylogenetic tree of the isolate

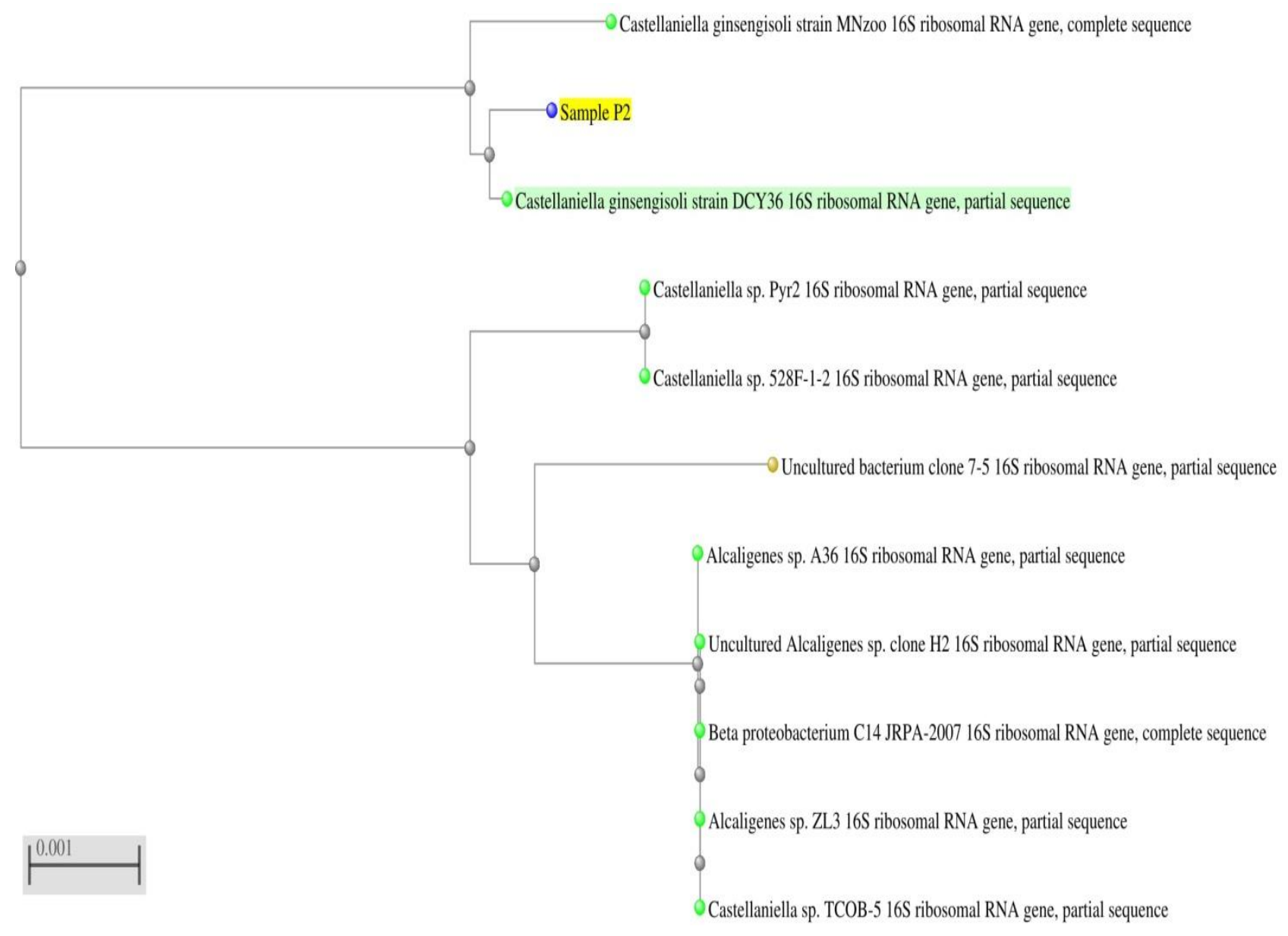


Fig.2 Secondary structure of rRNA of the isolate

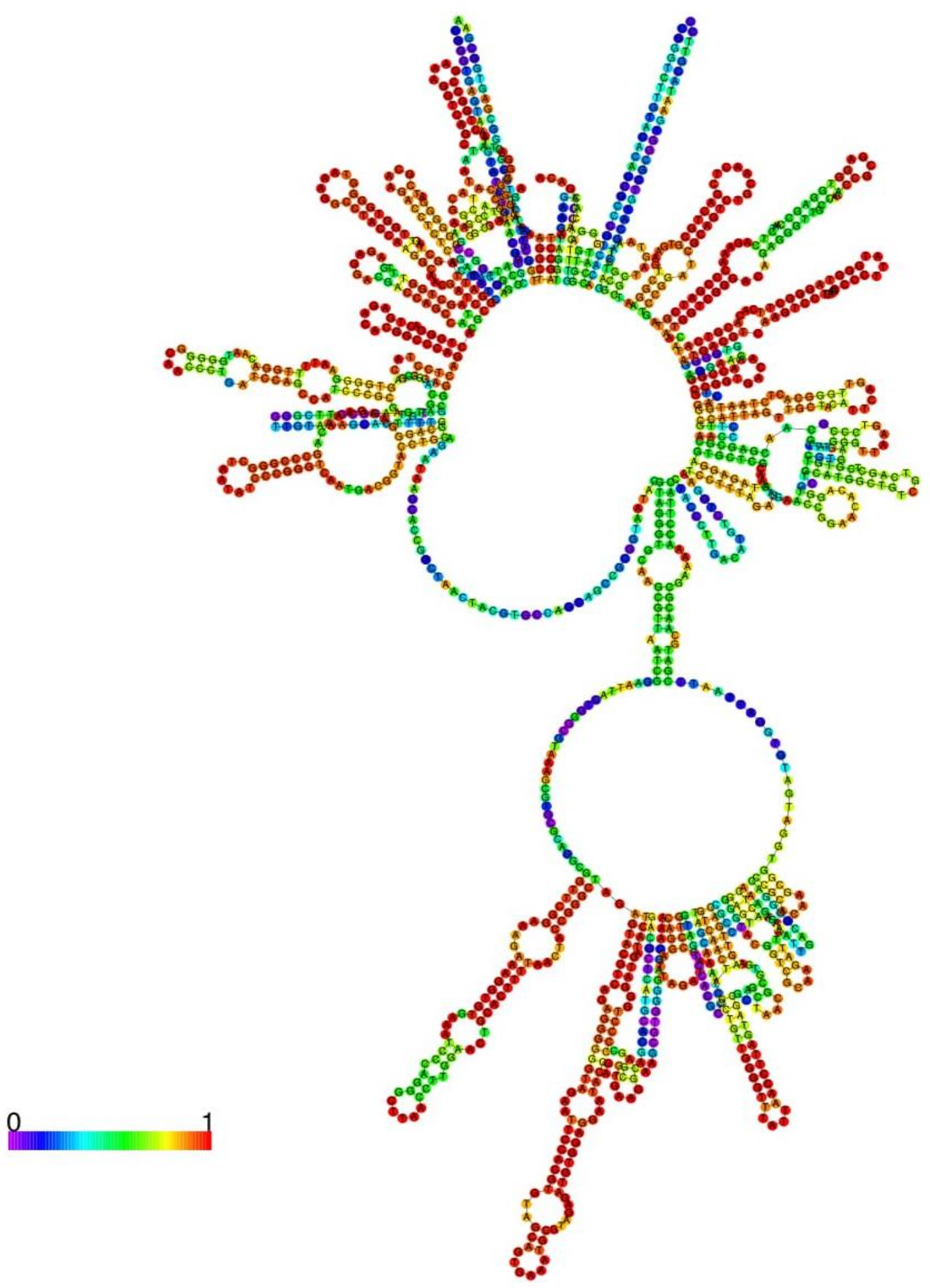


Fig.3 Mountain plot of secondary structure of rRNA of the isolate, is representation of the minimum free energy (MFE) structure, the thermodynamic ensemble of RNA structures, and the centroid structure and the positional entropy for each position

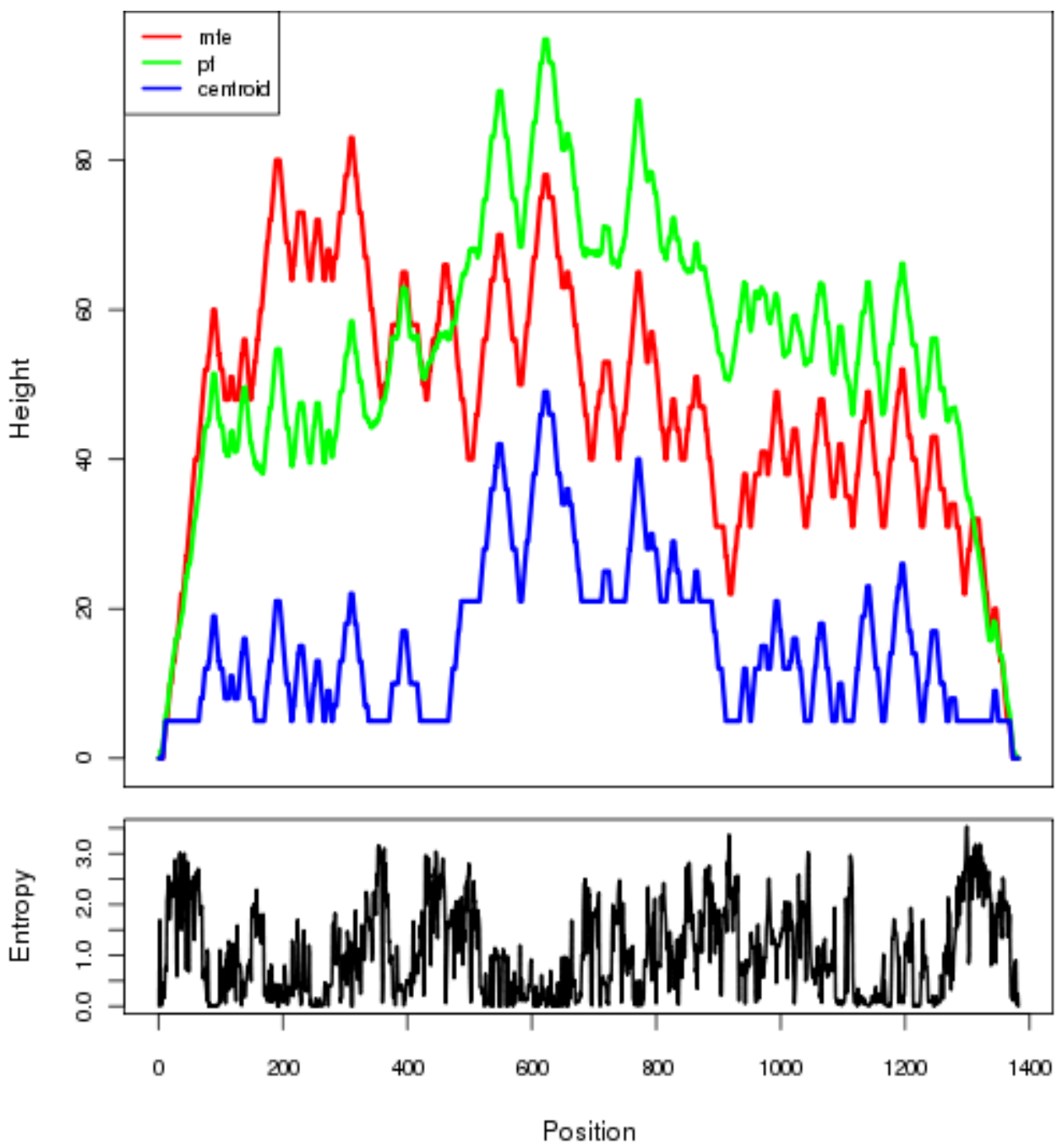

In order to understand the significance in predicting the stability of chemical or biological molecules or entities of Castellaniella ginsengisoli, RNA secondary structure prediction has been performed. The 16S RNA gene sequence obtained was used to deduce the secondary structure of RNA ViennaRNA Web Services (Figure 2). This server provides programs, web services, and databases, related to our work on RNA secondary structures. For thermodynamic structure prediction, RNAfold web server was used. The RNAfold web server will predict secondary structures of single stranded RNA or DNA sequences and current limits are 7,500 nt for partition function calculations and 10,000 nt for minimum free energy only predicitions. The free energy of secondary structure rRNA of Castellaniella ginsengisoli was $-505.30 \mathrm{kcal} / \mathrm{mol}$. Mountain Plot was also drawn by the RNAfold web server which helps in predicting the hierarchical organization of RNA secondary structure, as nested helices translate into stacking mountains, easing the visual segmentation into domain. Here the sequence is drawn linearly, but this representation also presents, at each position $i$, 
the number of base-pairs nesting the position, i.e. involving bases respectively before and after i. In this setting, helices give rise to mountains while terminal loops translate into peaks (Figure 2).

Castellaniella ginsengisoli is a Gramnegative, oxidase- and catalase-positive, rodshaped, motile, beta-glucosidase-producing bacterium from the genus Castellaniella which has been firstly isolated from soil of a ginseng field in South Korea (Kim et al., 2009). Colonies of Castellaniella ginsengisoli are yellow coloured. The genus Castellaniella was created by the reclassification of Alcaligenes defragrans DSM 12141T to the type species of the genus Castellaniella.

The genus contains these species, Castellaniella defragrans, Castellaniella denitrificans (Kampfer et al., 2006) and Castellaniella caeni (Liu et al., 2008), Castellaniella ginsengisoli (Kim et al., 2009) and Castellaniella daejeonensis (Lee et al., 2010) Castellaniella hirudinis. In 2006, the genus Castellaniella was proposed (Kampfer et al., 2006) for Gramnegative organisms characterized by short motile rods with a facultatively anaerobic and denitrifying metabolism. All species described so far contain ubiquinone Q-8 as the major quinone, phosphatidylethanolamine and phosphatidyl -glycerol as the major polar lipids and C16: 0, C16: 1v7c and C17: 0 cyclo as the major fatty acids (Kim et al., 2009).

In conclusion, molecular techniques utilizing polymerase chain reaction (PCR), alone or in combination with DNA sequence analysis, have become increasingly popular in determining the evolutionary relationships of bacteria which are helping in the identification of novel bacteria.

\section{References}

Singh, B.K., Milard P, Whitely AS, Murrell JC. 2004. Unravelling rhizosphere-microbial interactions: opportunities and limitations. Trends Microbiol 12: 386-393

Chandra, P., and Chandra. A., 2016. Elucidation of rRNA secondary structure and phylogenetic analysis of plant growth promoting Streptomyces sp. based on $16 \mathrm{~s}$ RNA. Journal of Wheat Research 8(2): 6165.

Pontes, D.S., Lima-Bittencourt, C.I., ChartoneSouza, E. et al., J Ind Microbiol Biotechnol 2007. 34: 463.

Arne, O., Mooers, and Stephen B. Heard, "Inferring Evolutionary Process from Phylogenetic Tree Shape," The Quarterly Review of Biology 72, no. 1 1997. 31-54.

Kim, M.K., Srinivasan S., Kim Y-J and Yang DC.Castellaniella ginsengisoli sp. nov., a bglucosidase-producing bacterium. International Journal of Systematic and Evolutionary Microbiology 2009. 59, 2191-2194

Kampfer, P., Denger, K., Cook, A. M., Lee, S.-T., Jackel, U., Denner, E. B. M. \& Busse, H.-J. 2006. Castellaniella gen. nov., to accommodate the phylogenetic lineage of Alcaligenes defragrans, and proposal of Castellaniella defragrans gen. nov., comb. nov. and Castellaniella denitrificans sp. nov. Int J Syst Evol Microbiol 56, 815-819.

Liu, Q. M., Ten, L. N., Im, W. T. \& Lee, S. T. 2008. Castellaniella caeni sp. nov., a denitrifying bacterium isolated from sludge of a leachate treatment plant. Int J Syst Evol Microbiol 58, 2141-2146.

Lee, M., Jung, H.-M., Woo, S.-G., Yoo, S.-A. \& Ten, L. N. 2010. Castellaniella daejeonensis sp. nov., isolated from soil. Int J Syst Evol Microbiol 60, 2056-2060.

\section{How to cite this article:}

Priyanka Chandra and Amaresh Chandra. 2017. Identification and Molecular Characterization of Castellaniella ginsengisoli Isolated from Sugarcane-Wheat Cropping System. Int.J.Curr.Microbiol.App.Sci. 6(10): 3509-3515. doi: https://doi.org/10.20546/ijcmas.2017.610.414 